\title{
Hydrogen Bonds in Polymer Folding
}

\author{
Jesper Borg, Mogens H. Jensen, Kim Sneppen and Guido Tiana \\ Niels Bohr Institute and Nordita, Blegdamsvej 17, DK-2100 Ø, Denmark
}

\begin{abstract}
The thermodynamics of a homopolymeric chain with both Van der Waals and highly-directional hydrogen bond interaction is studied. The effect of hydrogen bonds is to reduce dramatically the entropy of low-lying states and to give raise to long-range order and to conformations displaying secondary structures. For compact polymers a transition is found between helix-rich states and low-entropy sheet-dominated states. The consequences of this transition for protein folding and, in particular, for the problem of prions are discussed.
\end{abstract}

PACS: 05.70.Jk,82.20.Db,87.15.By,87.10.+e

Secondary structures are a prime feature of all structured polymers and proteins [1] 2]. These structures are stabilized by hydrogen bonding. For example, $\alpha$-helices of proteins are known to be stabilized by hydrogen bonds which involve couples of donor and acceptor atoms belonging to consecutive turns of the helix [2]. Similarly, hydrogen bonds are responsible for stabilizing the helix conformation in the helix-coil transition in amino acid homopolymers [3]. Recently, there has been a wide developement of simplified lattice models for protein folding, where each monomer interacts with its neighbours through an isotropic Van der Waals interaction [1]. These studies, in general, show no evidence of secondary structure formation for chains of realistic length and mostly deal with statistical features of good folders [9, 10].

In this paper we discuss the effect of including directed hydrogen bindings in simplified polymer models. As a starting point we adopt a lattice implementation widely used in the literature [5] 8]. This model is defined by a string of monomers placed on subsequent positions of a 3 -d cubic lattice. The energy of a configuration $\left\{\mathbf{r}_{i}\right\}$ is defined by the Hamiltonian

$$
H_{V W}=-\sum_{i<j} \epsilon_{i j} \delta\left(\left|\mathbf{r}_{i}-\mathbf{r}_{j}\right|-1\right)
$$

where $\sum$ include all monomer pairs, the $\delta$ function ensures contributions from nearest neighbours only and $\epsilon_{i j}$ contains the strength of Van der Waals interactions. In the case of homopolymers, all $\epsilon_{i j}$ are equal, while for proteins different choices can be adopted [7, 1, 11].

In addition to $H_{V W}$, we introduce in the Hamiltonian a term associated with a directed interaction between couples of monomers. On each monomer $i$ we assign a spin $\mathbf{s}_{i}$ representing a hydrogen donor-acceptor pair. This can be easily pictured as a spin because of the opposite directions of the $O(\mathrm{H}$ acceptor) and the $N$ (H donor) atom on the peptide backbone. The spin is constrained to be perpendicular to the backbone. To study the secondary structure of proteins, donors and acceptors coming from the amino acid sidechains should be taken into account as well. In this simple model we consider the minimal scenario of a homopolymer with only one acceptor and one donor for each monomer. The hydrogen bond part of the Hamiltonian reads

$$
H_{H}=-\epsilon_{H} \sum_{i j} \delta\left(\mathbf{s}_{i} \cdot \mathbf{s}_{j}-1\right) \delta\left(\left|\mathbf{r}_{i}-\mathbf{r}_{j}\right|-1\right),
$$

where only $\mathbf{s}_{i}$ that are perpendicular to the backbone are allowed, and thus interactions along the backbone are automatically ignored.

Setting $\epsilon_{i j}=\epsilon_{V}$ for all $i$ and $j$, the Hamiltonian $H\left(\epsilon_{V}, \epsilon_{H}\right)=H_{V W}+H_{H}$ specifies the energy of any homopolymer configuration including hydrogen bindings. To study the equilibrium properties we sample configurations of the chain using the simulated tempering techniques suggested by Marinari and Parisi [12] and developed in the context of proteins by Irbäck and Potthast [13]. This method consists in examining an ensemble of different temperatures, by sampling a generalized partition function that includes the temperature as a dynamical variable. By adjusting the sampling rate associated with each temperature, one avoids trapping in local minima. The sampling rates are adiabatically adjusted according to the multiple histogram equations [14 and, to avoid overestimation of metastable states, we test for thermodynamic compatibility as described in ref. 15.

Figure 1 shows examples of structures obtained at low temperatures using $H(0,2)$ and $H(1,2)$, respectively. In the first case, one can observe helix-like structures that, however, differ from true $\alpha$ helices by the change in chirality along the helix. This is due to lattice constraints [15]. We denote this kind of structures pseudo-helices ("p-helix"), and quantify them for a given polymer conformation by counting the number of bonds involved in them. 
For example, in Fig. 1(a) the monomers between 2 and 7 initiate a pseudo helix, where neighbours $2-5$ and neighbours 4-7 contribute. The p-helix continues until monomer 22 , which breaks it because this monomer is not neighbour to any members of a $\mathrm{p}$-helix. A new pseudo-helix is initiated at monomer 25 and last throughout the chain. A helix is similarly defined as a structure built as that of Fig. 1(a), but with constant chirality (it would have been the case if the position of monomer 7 and 9 were interchanged in Fig. 1(a)).

Fig. 1(b) displays the ground state of a polymer where Van der Waals interactions are included. In this case one can observe structures resembling $\beta$-sheets. The sheets can be either parallel or antiparallel, as in natural proteins, in both cases quantified by identifying at least three pairs of consequtive neighbours in a line (that is for parallel sheet it would be $\{(i, j),(i+1, j+1),(i+2, j+2)\}$ and for antiparallel sheet $\{(i, j),(i+1, j-1),(i+2, j-2)\})$. In Fig. 1 (b), for example, monomer pairs $(1,10),(2,9),(3,8)$ and $(4,7)$ contribute to an antiparallel sheet, that gets broken at monomer 10. Monomers 6-13 also participate in an antiparallel sheet with the layer above.

Both the folds displayed in Fig. 1 reveal long range order. In particular, Fig. 1(b) displays an up-down symmetry and an organization where sheets are on one side of the structure and the backbone connections between layers are concentrated on the opposite side. The key-result of this analysis is, in fact, that spin interactions induce large scale organization even in the case of a homopolymer collapse.

Furthermore, the presence of hydrogen bonds causes a dramatic reduction of entropy, compared to that found for homopolymers with only isotropic interactions. The conformation displayed in Fig. 1(b) is, in fact, the unique, zero entropy ground state for a 36mer interacting through the Hamiltonian $H(1,2)$ (except for trivial symmetries, i.e. lattice symmetries and flipping of lines of spins. The latter being easily removed by introducing a diedral term in the Hamiltonian). The reduction in entropy can be appreciated from the inset to Fig. 2.

Fig. 2 also displays the number of bonds involved in the four types of secondary structures $(I)$, as function of total number of bonds $\left(N_{B}\right)$. The dependence with number of bonds is obtained by thermal averaging as function of temperature. The choice of using $N_{B}$ rather than the temperature as free variable is more convenient, since we are comparing systems with different energy scales. The three curves represent the case where only Van der Waals energy is present $\left(\nu \equiv \epsilon_{H} / \epsilon_{V}=0\right.$, full line), where Van der Waal and spin coupling energy are present $(\nu=2)$, and the case where only spin coupling is present $(\nu=\infty)$. For any degree of compactness the amount of secondary structure increases with increasing spin coupling. In general, it also increases with compactness, however a backbending for the $\nu=2$ case is present at nearly maximal compactness. The backbending for $\nu=2$ in both plots is the mark of a phase transition. The transition takes place at almost maximum compactness (which is associated with low temperature), where entropy is reduced abruptly while compactness changes to a minor extent. In fact, this transition distinguishes between two types of compact polymers, a phase in which p-helices are predominant, and an ordered, highly symmetric one, rich in $\beta$-sheets (cf. Figs. 1b,3).

Fig. 3 shows in detail how the different types of secondary structures change with respect to temperature $T=1 / \beta$. The backbending in Fig. 2 corresponds to the transition at $\beta=3 / \epsilon_{V}$, in which relatively disordered states with a large fraction of pseudo-helices are replaced with ordered, sheet-dominated structures like that displayed in Fig. 1b. We notice that even the conformations at intermediate values of the temperature $\left(\beta\right.$ in the range between $1 / \epsilon_{V}$ and $\left.2.5 / \epsilon_{V}\right)$ are quite ordered, in the sense that they have a large degree of helix structures. This behaviour can be compared with the case of homopolymers without spin interactions, which have significantly less structures, and in particular have much lesser helix content (data not shown). The conformations mentioned above are rather compact, the homopolymer collapse taking place at $\beta \sim 1 / \epsilon_{V}$, while the helix-sheet transition takes place at $\beta \sim 3 / \epsilon_{V}$. The gap between the two transitions are adjustable by changing $\nu$, f.ex. for $\nu=3$ we find the two transitions much closer to each other.

Most interestingly, the energy landscape of even the simple homopolymer turns out to be extremly rough, so rough that the helix-sheet transition cannot be sampled with any normal Metropolis approach. In other words, a polymer with hydrogen bonds displays a population of low energy conformations which are structurally very different from each other, separated by high energy barriers and resembling rather closely the spin glass behaviour [16].

It is remarkable that the present model predicts a large variety of helix structures, contrasting to a few, highlyordered sheet structures. This fact suggests that helix-like conformations are entropically favoured in the protein folding mechanism, and consequently can act as intermediates leading the chain to its equilibrium state, while sheetlike structures first appear late in the folding process and contribute to the stabilization of the ground state. A similar pattern is found in the study of prion diseases [17], where the sane, helix-dominated native form of the protein seems to be a very long-lived metastable easily accessible state, whereas the true ground state is dominated by $\beta$-sheets and prone to aggregation.

Finally, we would like to stress that the current parametrization of hydrogen bonding is the simplest possible one. The driving force in protein folding is believed to be hydrophobicity, where non-polar amino acids are deficient in hydrogen bonds and thereby cause an ordered, entropically unfavourable arrangement of the hydrogen bonds in the 
surrounding water. On the contrary, hydrophilic amino acids contain hydrogen receptors and donors that can replace those that water cannot build due to the presence of the protein surface. Thus, a more realistic model should explicitly include water. To model protein realistically, one should also consider chains composed of monomers with different amount of hydrogen donors and acceptors, reflecting the different types of amino acids. In such a way one will be able to control the sequence of secondary structures in a more specific way than with heterogeneous Van der Waals forces alone.

In summa, we have implemented a minimal model to keep into account hydrogen bond effects in polymer folding. Already at the level of homopolymers we have observed pronounced secondary structures, structures which are ubiquitous in the realm of natural proteins. 
FIG. 1. (a) A typical low energy structure for the homopolymer when only spin coupling is included. The different gray scales of the monomers are only meant to help the visualization of the 3-D structure. (b) The ground state when both spin and isotropic Van der Waals interactions are included $(\nu=2)$.

FIG. 2. Total number of nearest neighbours, $I$, involved in the four types of structures, as function of total number of nearest neighbours $N_{b}$ (i.e., as function of compactness). One can observe that the more hydrogen bonding (i.e. higher $\left.\nu=\epsilon_{H} / \epsilon_{V}\right)$ the more secondary structure is present and, as revealed by the inset, the less entropy $\Delta S=S(T)-S(T=\infty)$ is displayed by the system. Furthermore, one notices that a too large hydrogen bond energy prevents the formation of a compact state, and thereby of an ordered ground state. At intermediate values of $\nu$, there appears a highly ordered ground state that is sharply separated from a disordered compact state by a structural transition.

FIG. 3. Number of bonds involved in various structures, $N_{i}$, as function of $\beta=1 / T$ for $\nu=2$. The curves refer to antiparallel sheets ("a-sheet", solid line), pseudo-helices ("p-helix", dashed line), helices ("helix", dot-dashed line) and parallel sheets ("sheet", dotted lines). One can observe two transitions, one at low $T$, associated with the melting of the sheet-dominated structure (cf. Fig 1(b)), and one at high $T$ (i.e. low $\beta$ ), associated to the collapse transition of the polymer. At intermediate $T$ helices are important, serving as intermediates for polymer folding, by reducing the temperature.

[1] L. Pauling, R.B. Corey and H.R. Branson, PNAS 37 1951, 729-740.

[2] B. Alberts et. al., "Molecular Biology of the Cell", Garland Publishing, New York 1994.

[3] H.A. Scheraga, Pure and applied Chemistry 36 (1973) 1.

[4] S. Miyazawa and R. Jernigan, Macromolecules, 18, 534 (1985)

[5] P. J. Flory, J. Chem. Phys 17, 303 (1949)

[6] N. Go, Int. J. Peptide Prot. Res. 7, 313 (1975)

[7] K. F. Lau and K. Dill, Macromolecules 22, 3986 (1989)

[8] E. I. Shakhnovich and A. M. Gutin, J. Chem. Phys., 93, 5967 (1989)

[9] B. Derrida, Phys. Rev. Lett. 45 (1980) 79

[10] J. Bryngelson et. al. Proteins 21 (1995) 167.

[11] H. Li, C. Tang, and N. Wingreen, Phys. Rev. Lett. 79 (1997) 765.

[12] E. Marinari and G. Parisi, Europhys.Lett. 19 (1992) 451-458

[13] A. Irbck and F. Potthast, J. Chem. Phys. 103 (1995) 10298-10305

[14] A.M. Ferrenberg and R.H. Swendsen, Phys. Rev. Lett. 61 (1988) 2635; A.M. Ferrenberg and R.H. Swendsen, Phys. Rev. Lett. 63 (1989) 1195.

[15] J. Borg, to be published (2000).

[16] M. Mezard, G. Parisi and M. Virasoro, Spin Glasses and beyond, World Scientific, New York, 1988)

[17] P. M. Harrison, P Bamborough, V. Daggett, S. B. Prusiner and F. E. Cohen, Curr. Opin. Struct. Biol. 7, 53 (1997) 


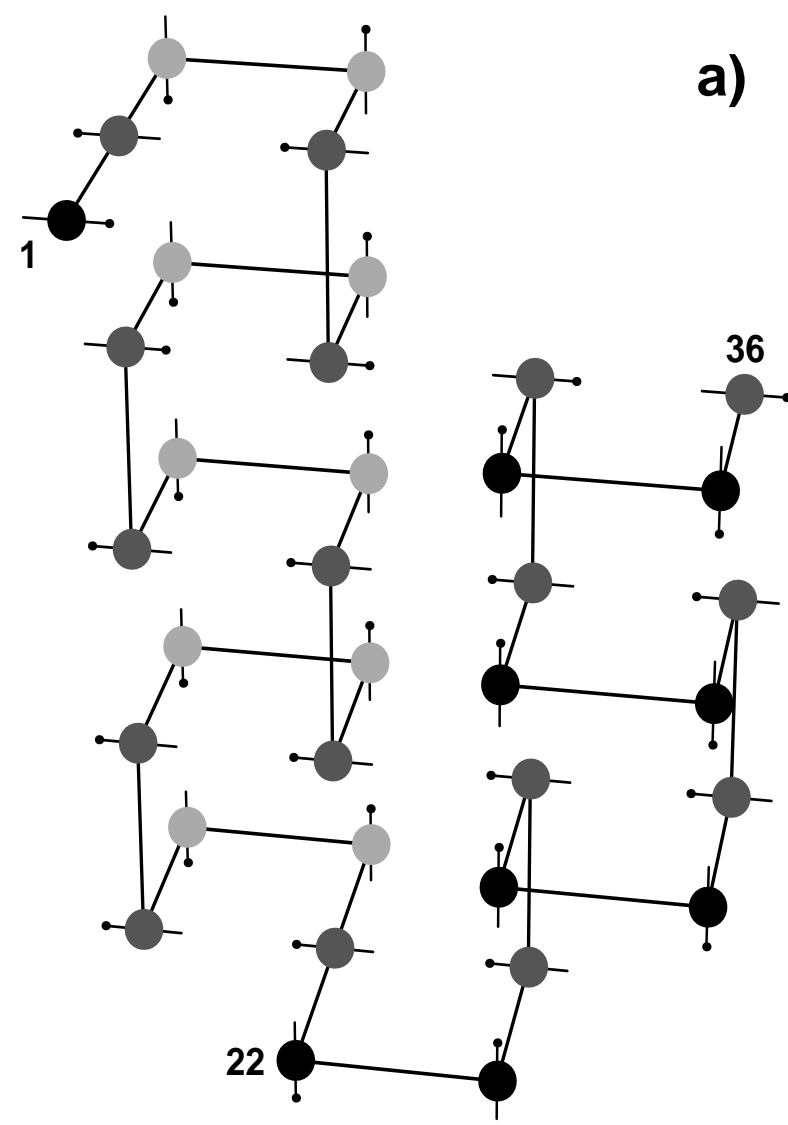

a)

b)

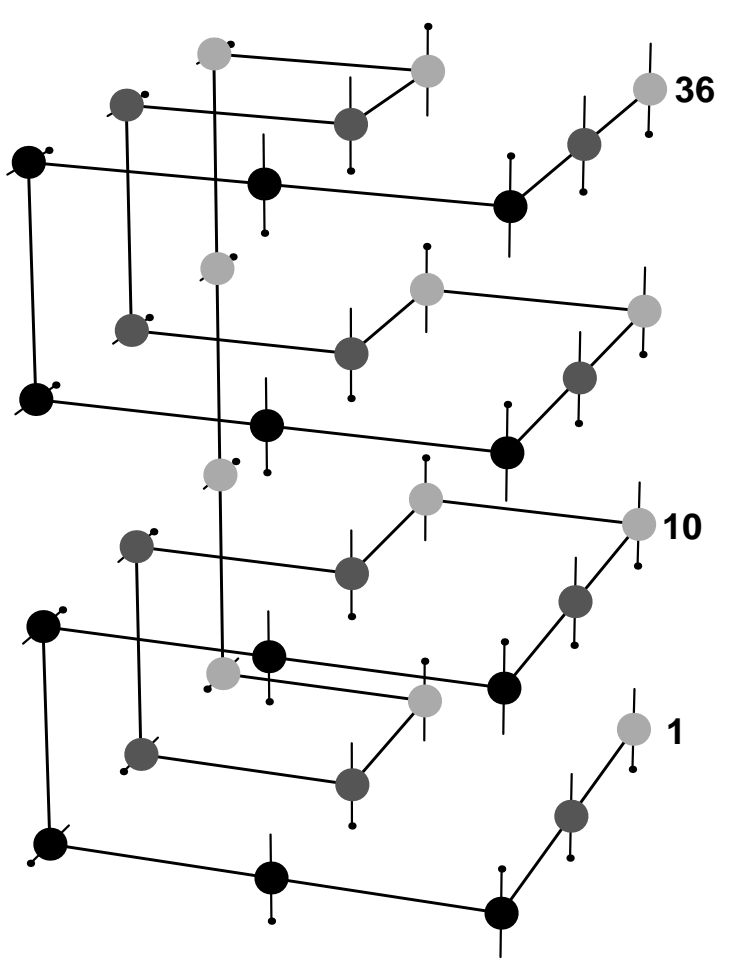




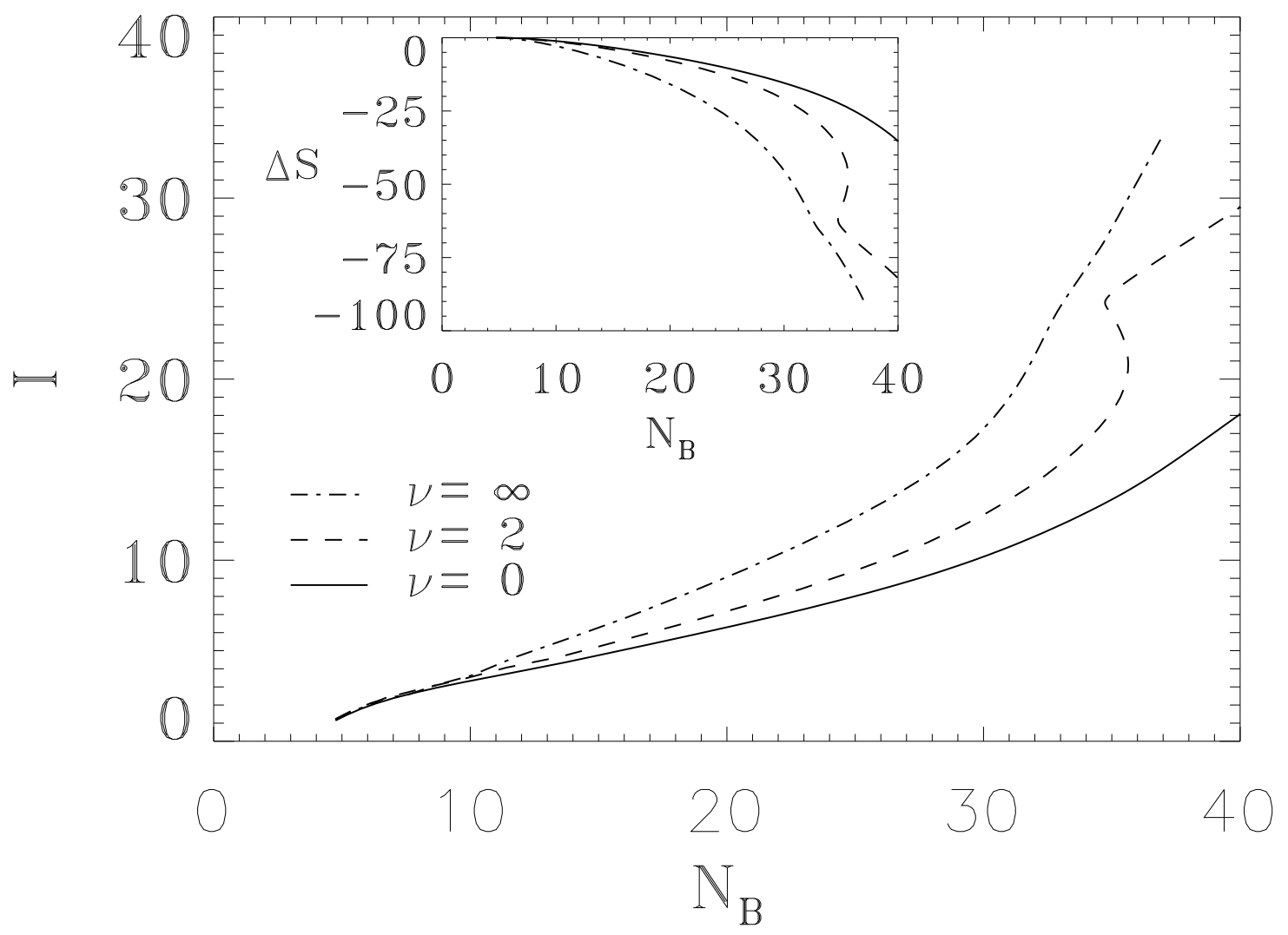






